Research

Article

\title{
Autonomous Learning System Towards Mobile Intelligence
}

\author{
Mengwei Xu (徐梦炜 $)^{1,2}$, Yuanqiang Liu (刘渊强 $)^{1,2}$, Kang Huang (黄康 $)^{3}$, \\ Xuanzhe Liu (刘譞哲) ${ }^{1,2}$, Gang Huang (黄罡) ${ }^{1,2}$ \\ 1 (Institute of Software, School of Electronics Engineering and Computer Science, Peking University, \\ Beijing 100871, China) \\ 2 (Key Laboratory of High Confidence Software Technologies of Ministry of Education, Peking University, \\ Beijing 100871, China) \\ 3 (Linggui Tech (Beijing) Co. Ltd., Beijing 100094, China) \\ Corresponding author: Xuanzhe Liu, xzl@pku.edu.cn
}

\begin{abstract}
How to efficiently deploy machine learning models on mobile devices has drawn a lot of attention in both academia and industries, among which the model training is a critical part. However, with increasingly public attention on data privacy and the recently adopted laws and regulations, it becomes harder for developers to collect training data from users and thus they cannot train high-quality models. Researchers have been exploring approaches to training neural networks on decentralized data. Those efforts will be summarized and their limitations be pointed out. To this end, this work presents a novel neural network training paradigm on mobile devices, which distributes all training computations associated with private data on local devices and requires no data to be uploaded in any form. Such training paradigm is named autonomous learning. To deal with two main challenges of autonomous learning, i.e., limited data volume and insufficient computing power available on mobile devices, this paper designs and implements the first autonomous learning system AutLearn. It incorporates the cloud (public data and pre-training)-client (private data and transfer learning) cooperation methodology and data augmentation techniques to ensure the model convergence on mobile devices. Furthermore, by optimization techniques such as model compression, neural network compiler, and runtime cache reuse, AutLearn can significantly reduce the on-client training cost. Two classical scenarios of autonomous learning are implemented based on AutLearn, with a set of experiments carried out. The results show that AutLearn can train the neural networks with comparable or even higher accuracy compared to traditional centralized/federated training mode with privacy preserved. AutLearn can also remarkably cut the computational and energy cost of neural network training on mobile devices.
\end{abstract}

Keywords machine learning; mobile computing; edge computing; distributed system

Citation Xu MW, Liu YQ, Huang K, Liu XZ, Huang G. Autonomous learning system towards mobile intelligence, International Journal of Software and Informatics, 2021, 11(2): 149-168. http://www.ijsi.org/ 1673-7288/00247.htm

This is the English version of the Chinese article “面向移动终端智能的自治学习系统. 软件学报, 2020, 31(10): 3004-3018. doi: 10.13328/j.cnki.jos.006064".

Funding items: Science Fund for Distinguished Young Scholars of China (61725201); R\&D Project in Key Areas of Guangdong Province of China (2020B010164002)

Received 2020-02-07; Revised 2020-04-04; Accepted 2020-05-09; IJSI published online 2021-06-22 
In recent years, machine learning technology represented by neural network has been rapidly developed, which has improved the intelligence of computer system and been widely applied in many important research fields such as computer vision and natural language processing. One of the development trends in machine learning applications is the gradual transfer from the cloud to mobile devices such as smartphones. For example, a recent empirical study ${ }^{[1]}$ found that the number of mobile applications equipped with neural networks and performing local computing in the Google Play APP store increased by $27 \%$ in the third quarter of 2018 . These deep learning applications have tens of millions of user downloads and comments, accounting for more than $10 \%$ of the total number of downloads and comments of all APPs in the APP store. In addition, major Internet manufacturers have self-developed deep learning frameworks for mobile, including Google TF Lite, Facebook Caffe2, Apple Core ML, Qualcomm SNPE, and Tencent ncnn, to optimize the deployment of neural network models on these resourceconstrained devices. Considering the performance advantages of neural networks and the more challenging deployment, this paper will take neural networks as a representative to research machine learning deployment issues on mobile devices.

One of the core challenges of developing a mobile-oriented neural network is that the model training requires a large amount of data which often comes from the mobile devices and contains a large amount of user private information. For example, the word prediction task of the input method application requires the input text of the user as the training set, which may contain the user's credit card number, chat history, email, and other private information. In recent years, various national institutions have introduced relevant laws and regulations to protect user data privacy in a more standardized way. For example, in 2018 the European Union formally implemented the General Data Protection Regulation (GDPR) ${ }^{[2]}$, which details the principles companies need to follow when it comes to user data. China formulated the Personal Information Protection Law, which legally defines the illegal collection of user information by APPs to protect user privacy based on laws.

How to train machine learning models on the premise of protecting user privacy is gradually attracting widespread attention in academia and industries. Technologies represented by federated learning ${ }^{[3]}$, homomorphic encryption ${ }^{[4]}$, and differential privacy ${ }^{[5]}$ give solutions to some extent, but they all have certain limitations. For example, federated learning distributes data and training on different mobile devices, and distributes models and aggregates gradients through a centralized parameter server to protect privacy. In the process of federated learning, however, there are still unsolved problems. For example, the upload gradient can beat tacked and the network bandwidth is costly. Homomorphic encryption uses classic cryptographic algorithms to protect data, but it will cause an extra additional computational cost, making it impossible to use in complex neural networks.

Therefore, this paper proposes a neural network training mode of "autonomous learning": The model calculation tasks related to user data are fully deployed in the local mobile to ensure that the data will not be transmitted to the outside in any form (raw data, encrypted data, and model gradient). This paper focuses on the model training process. Compared with the traditional centralized or federated learning, in addition to protecting user privacy to a greater extent, autonomous learning has two other advantages. One is personalized learning. Autonomous learning uses the data generated on each mobile to train its own model, and then serves the specific user, which means that the model is formed by personalizing training and learning for each user. In many mobile scenarios, the behavior habits of users (such as input methods and voice assistants) are different. The personalized training model has a higher accuracy rate than the global model shared by all users. The other is high scalability. The hardware resources required by the traditional centralized/federated learning mode, including 
computing, storage, and network bandwidth, show an approximate linear relationship with the number of mobile devices accessed. For applications with a large number of users, this will greatly increase the cost of application development and maintenance. However, autonomous learning requires that each access device only conducts model training through local computing storage resources, and the cloud does not need to provide more hardware resources, so it has higher scalability.

There are two main challenges in implementing an autonomous learning system. Firstly, how to get high-quality neural network models by training without the mobile data being transmitted to the outside world? Secondly, due to the limited hardware resources on each mobile device, how to quickly complete the training and deployment without affecting user experience? In order to solve these two problems, we design and implement the first mobileoriented autonomous learning system. The core idea of AutLearn is as follows: With the cloud-client cooperation methodology, public training data are used to train a model of a strong generalization ability in the cloud server; transfer learning technology to adjust the model at the mobile to obtain a model that is suitable for the mobile device and can be used for deployment. Pre-training in the cloud adopts public non-private data sets without requiring users to upload any data. On-device transfer learning uses the data augmentation techniques to improve the training effect. In order not to affect user experience while using mobile devices, the on-device transfer learning is further divided into two modes: mobile offline learning and mobile online learning, so as to suit different training scenarios and objectives. In addition, AutLearn introduces a number of optimization techniques, including model compression, runtime cache optimization, and neural network compiler. These techniques can greatly save the resource cost of neural network training at mobile devices at little loss of accuracy.

We implement two typical application scenarios of autonomous learning based on AutLearn: input word prediction and image classification, and verify them on large-scale data sets and classical neural network models. The experimental results show that AutLearn can get a neural network model at high accuracy by training on the premise of protecting user privacy. Compared with the centralized/federated training mode, the model obtained by autonomous training can achieve similar or even higher accuracy, resulting in personalization. In addition, mobile training optimization technology of AutLearn can at most save over $80 \%$ of the trainable time and mobile device energy consumption. Combined with our observations of the behavior of real mobile users, AutLearn can train new model for deployment in one day.

\section{Background and Related Work}

\subsection{Background}

Machine learning technology represented by deep learning algorithms has received extensive attention from academia and has also been greatly deployed in industries. The quality of deep learning models depends on the training data whose quantity and quality determine whether the accuracy of the final training model meets the requirements. However, these data often need to be collected from the user device and contain the private information of users. Once leaving the mobile device, the data will bring the risk of privacy leakage. For example, the word prediction task of the input method application requires the input text of the users as the training set, which may contain the credit card number, chat history, email, and other private information of users. When the data are uploaded to the server, the traditional information security technology, such as encrypted transmission and anonymous user, cannot guarantee that the user data will not be leaked and used maliciously. In order to protect user data in a more standardized way, various national agencies have introduced relevant laws. For example, in 2018 the European Union formally implemented the GDPR ${ }^{[1]}$, which details the principles 
companies need to follow when it comes to user data. How to train deep learning models with high quality on the premise of protecting user privacy is a challenge. Therefore, researchers have proposed different solutions. Some of the main related work will be outlined and their shortcomings will be summarized in the next section.

\subsection{Related work}

The federated learning technology is originally proposed by Google AI team ${ }^{[3]}$. The core idea of federated learning technology is as following: Instead of uploading user data directly, the model training task is deployed to the mobile device, which uploads the updated model to the cloud after the training is completed, and the cloud only needs to update and aggregate the models from different mobile devices and issue the aggregated model. Since the relationship between the input data and the gradient is a many-to-many and high-dimensional space mapping, it is difficult for an attacker to obtain the original data according to the model gradient. Therefore, federated learning protects user privacy to a certain extent. A lot of follow-up work focuses on how to optimize the process of federated learning. For example, in order to reduce the communication cost from the mobile to the server, Konečný et al. ${ }^{[5]}$ proposed two optimization strategies: structured update and shorthand update. Structured update limits the model parameters to a smaller solution space, so that the model can be represented with fewer parameters to reduce the size of the model gradient. Shorthand update still uses the original model for training, but the model gradient obtained by training is compressed before uploading, for instance, by means of quantization and down sampling. In order to solve the heterogeneity of mobile devices in federated learning process, the SmartPC system proposed by Li et al.$^{[6]}$ adopted dynamic pacing synchronization strategy to dynamically adjust the current waiting period in each training round according to the delay of receiving the update of terminal model in the last round. This work also proposed to dynamically adjust the CPU operating frequency of mobile devices to ensure that the gradient can be uploaded before the ending of waiting period and minimize the training energy consumption. In order to further optimize the data security in the federated learning process, Bonawitz et al. ${ }^{[7]}$ proposed that the model updating was fused at the mobile through the security multi-party computation technology, so as to ensure that the server could only see the overall update after fusion and could not infer its training data through the model updating of the individual.

The homomorphic encryption algorithm allows the encrypted result of performing algebraic operation on ciphertext. The result obtained by decrypting ciphertext is consistent with that of decrypting plaintext. In other words, this technology allows us to perform operations such as retrieval and comparison in the encrypted data to get the correct results, and there is no need to decrypt the data during the entire process. Some related works ${ }^{[8,9]}$ have explored how to apply homomorphic encryption to the machine learning training, but the application mainly concentrated on some simple algorithms, such as support vector machines and decision trees. Due to the complexity of deep learning operations, designing homomorphic encryption algorithms for them has greater challenges. In the meantime, training on encrypted data is also far more complicated than prediction ${ }^{[10]}$. Nandakumar et al. ${ }^{[11]}$ initially discussed and realized how to use homomorphic encryption technology to perform deep learning training on encrypted data. They used ciphertext packing technology to accelerate training, but the training efficiency is still not high, so that the convolutional layer operation cannot be supported and there is even a small accuracy loss of the training model.

Differential privacy ${ }^{[12]}$ is originally designed for database query. Its purpose is to quantify user privacy, ensuring that users can only obtain statistical information from the database, and cannot obtain individual information. It maximizes the accuracy of data query. The specific 
implementation of differential privacy is mainly to add randomness to the query results, such as noise that follows Laplace distribution and exponential distribution. In order to apply differential privacy technology to data protection during machine learning training, Abadi et al. ${ }^{[5]}$ proposed the training algorithm of modifying the model, that is perturbation is added to the gradient obtained from training the data of each batch, and then the perturbed gradient is applied to the model training. In this process, the numerical value of the perturbation can also be accumulated, so as to obtain the quantification of the overall privacy after the training. This technology has also been integrated into the TensorFlow open source project to help developers train deep learning models with privacy protection. Shokri et al.$^{[13]}$ proposed a strategy of partial sharing gradient, namely after each round of training, part of the gradients are selectively shared, and then the gradients of multiple organizations are aggregated and applied to the old model parameters wholly to obtain the updated model. The main reason why this strategy is effective is that the gradient descent training has strong robustness to unreliable model gradients with random properties. With this strategy, Shokri et al. further used differential privacy technology to quantify and reduce the risk of privacy leakage.

Summary: Although the above technologies can protect user privacy to a certain extent, they also have limitations. For example, federated learning technology always requires users to upload data-related information (model gradient or model itself), which leads to the risk of privacy leakage ${ }^{[14]}$. In addition, federated learning will lead to a large amount of network communication cost, since it has high requirement for bandwidth. Homomorphic encryption technology will cause a large amount of computational cost, and cannot be applied to complex neural network structures and practices. Differential privacy technology mainly protects the data information in the model obtained after training, but it cannot protect the training data from being obtained and used maliciously during the training.

\section{Distributed Autonomous Learning: the Analysis of Advantages, Challenges, and Feasibility}

This paper proposes the concept of distributed autonomous learning. The core idea is that mobile devices do not need to upload user data in any form, and all operations (model training) related to user data are performed locally. To a certain extent, autonomous learning can be regarded as a special case of the development direction of centralized learning to the federated learning in recent years, that is, uploading raw data (centralized learning) $\rightarrow$ uploading training result data (federated learning) $\rightarrow$ uploading no data (autonomous learning). The degree of privacy protection is also increasing progressively. In autonomous learning, each client device does not rely on any external data and computing power. Starting from an initial model, a new locally deployed personalized model with high precision is obtained through local data and computing power.

This autonomous learning computational model is also in line with the decentralized idea that has gradually attracted attention in recent years. Although federated learning has been decentralized to a certain extent (compared to centralized learning), there is still a centralized node which is used to coordinate the computational tasks of each mobile and summarize the model parameters uploaded by these mobiles. However, autonomous learning achieves a machine learning training model which is more thoroughly decentralized.

We believe that the advantages of autonomous learning are mainly reflected in the following three aspects.

- The protection of user privacy: Since the mobile device does not upload any data, all data are generated, stored, and consumed on the local mobile device, which greatly reduces the risk of privacy leakage. Correspondingly, federal mechanisms still need to upload 
user data that is indirectly related to user privacy (such as model updating gradients), so it still has the risk of privacy leakage. From the perspective of the end user, keeping the data locally is obviously easier to accept and is more conducive to the implementation of data protection regulations in various countries.

- Personalized model: The process of autonomous learning is to use the data generated on each mobile to train its own model. This model will be deployed to serve the specific user, which means that the model is obtained by personalized training and learning for each user. In many scenarios, the behavior habits of users (such as input methods and voice assistants) are different, so a globally unified global model trained from centralized or federal model cannot serve different users well. Personalized ability acquired by autonomous learning is needed to solve this problem.

- Higher scalability: Autonomous learning means that each newly-connected device will provide its own computational and storage resources for model training, and the cloud does not need to provide more hardware resources, so it has high scalability. The centralized/federated learning model requires the cloud to provide more hardware support for newly-connected mobile devices, including computing, storage, and network bandwidth. For applications with a huge number of users, this will be costly.

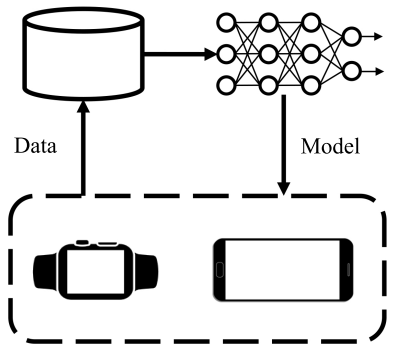

(a) Centralized learning

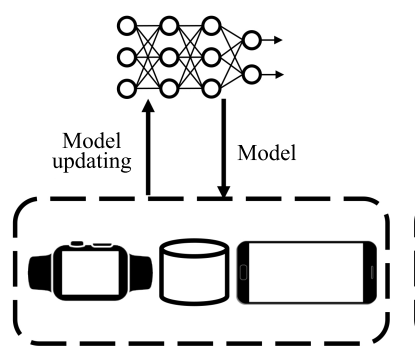

(b) Federated learning

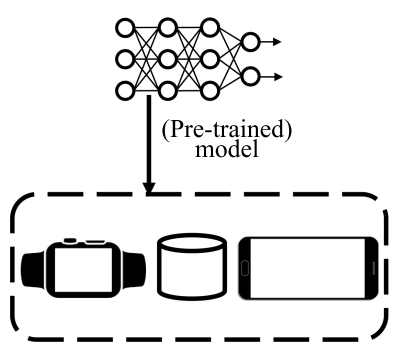

(c) Autonomous learning

Figure 1 Comparison between different machine learning training paradigms

In fact, the traditional view is that both user privacy and user personalization are difficult to achieve at the same time ${ }^{[15]}$, because the two are inherently contradictory. Autonomous learning solves this problem to a certain extent by making full use of the local computational power of the mobile device.

Challenges: The challenges of implementing an autonomous learning system mainly exist in two aspects: insufficient local data and terminal computational power of mobile device. First, training machine learning models, especially neural network models, requires a large amount of data. However, the training data on each mobile device is often limited. Training the model with insufficient data may cause the model to fail to converge, which lead to the failure of obtaining a high-quality model. It also requires considerable computational resources, but the computational power of mobile device is often limited. Considering the complex operating environment of the mobile device and the interaction among users, the mobile device cannot be used for model training at any time. A direct question is that when a new model structure is designed and distributed to each mobile device, how long will it take to complete training and use for deployment?

Feasibility analysis: Offline model training is the most direct way of autonomous learning, namely after the client obtains a new model structure from the cloud, the model is trained at an appropriate timing, and then it is used for deployment. Here, "appropriate" training timing mainly refers that the user experience will not be affected during this period. 
In most federated learning systems ${ }^{[16]}$, the model training will be carried out only when the following conditions are met: (1) The device is being charged. (2) The screen of the device is turned off (namely it is not used interactively by the user). (3) The device is in a non-chargeable network environment (such as WiFi). For autonomous learning, since there is no need to upload the model, condition 3 can be ignored. Even so, the remaining two harsh conditions still greatly limit the time that mobile devices can spend for offline training. We found that the existing work lacks relevant real user data to support this offline deep learning training mode. For this reason, we collected behavioral data of using smartphones of nearly 1,500 real users, including the transition of the hardware state (network state, charging state and screen state) on these devices.

Figure 2 illustrates the analysis results of user behavioral data. In this figure, the abscissa represents the trainable time that a single mobile can be used for model training per day, namely the trainable time that the above conditions of system state are met; the ordinate represents the Cumulative Distribution Function (CDF) of the trainable time on different devices and users. We found that the trainable time each user can use for autonomous training is about 120 minutes (median) per day, while the duration for federated learning is 60 minutes (median), because the requirements of federated learning for the device to participate in training is more harsh, namely the condition that the device is in a non-chargeable network environment must be met. We believe that 120 minutes is usually enough to complete a large number of model training tasks. Combining the AutLearn system and its optimization technology we propose below, it is sufficient to achieve the purpose of autonomous learning. We will specifically introduce the computational cost of autonomous learning on mobile devices in Section 4.3.

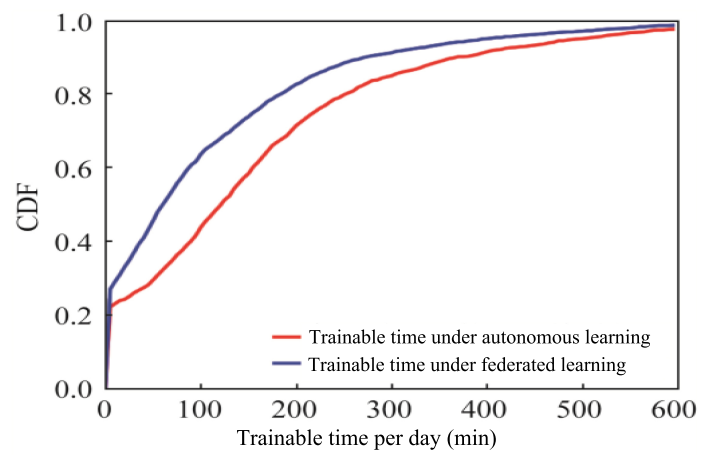

Figure 2 Trainable time per day under federated learning and autonomous learning

\section{Distributed Autonomous Learning System-AutLearn: Design and Implementation}

We have implemented the first distributed autonomous learning system, AutLearn. This section introduces the design and specific implementation of the AutLearn system. First, the core idea of the system is introduced; then, the overall architecture and the specific implementation of each functional module in the system are presented; finally, how specific applications run on the system to achieve autonomous learning is adopted with input method and image processing as examples.

\subsection{Core idea}

In order to solve the problem of insufficient training data on mobile devices, AutLearn adopts the idea of cloud-client cooperation methodology, uses public data on the cloud server to train a model with strong generalization ability, and then adjusts the model on the mobile by the 
transfer learning technology ${ }^{[17]}$ to obtain a model suitable for the mobile device and available for deployment. In order to ensure user privacy, the cloud server cannot require the user to upload data in model pre-training, but uses data mining to find the surrogate data which has similar distribution of user data from public data to achieve public-private win-win cooperation. The public data here include data that has been authorized by users, data purchased through legal channels, and data crawled from the public network via crawler technology. In addition, we have also introduced data augmentation techniques to increase the amount of data used for training on the mobile.

In order to solve the problem of limited computational resources and power of mobile device, AutLearn employs optimization techniques such as model layer compression, runtime cache optimization, and neural network compiler. These techniques greatly save the resource cost of neural network training on mobile device at little loss of model accuracy.

\subsection{Overall architecture}

The autonomous learning system framework, AutLearn, proposed in this paper is shown in Figure 3. On the cloud server, the public data set is first used to pre-train the model. Considering that the model needs to be deployed on the mobile, the AutLearn has a built-in model pruning algorithm based on singular value decomposition ${ }^{[18]}$. After the pruning, the model needs to be retrained to restore the model accuracy. Next, the AutLearn will use neural network compilation technology to compile the pruned model to optimize its computational efficiency on the corresponding mobile computing hardware (CPU, GPU). The specific content of compilation will be introduced in Section 3.4.

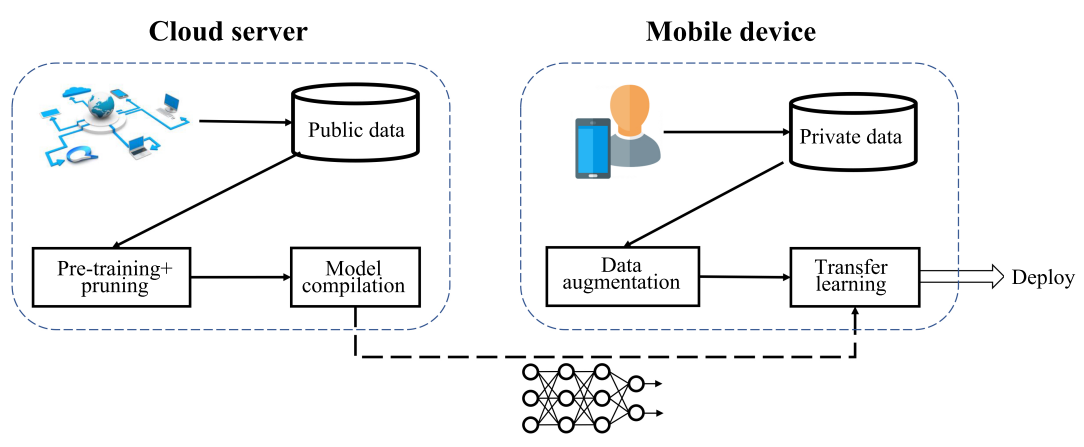

Figure 3 Architecture of AutLearn

The compiled model is downloaded to each mobile device, and the local data are used for transfer learning at an appropriate time period. This process is called offline learning. During the process, AutLearn will enhance the local data to increase the amount of data. After the training, the model will be used for deployment and perform forward inference of the neural network. In order to allow the model to continuously learn the changes of user behavior to obtain higher accuracy, AutLearn also implements online learning, namely immediately training data after the training data are generated on the mobile devices. Compared with offline learning, online learning can adapt to the changes of user behavior faster, so that more reasonable results can be obtained in the next inference, and the data does not need to be stored locally, further reducing the risk of privacy leakage. Specifically, whether to use offline learning or online learning, or a combination of the two, depends on the specific application scenario. We will introduce how to choose the appropriate training mode in Section 3.6. 


\subsection{Cloud-client cooperation training}

The purpose of cloud-client cooperation training is to use public data sets to obtain a model with strong generalization ability by training in the cloud, so that the model can be finely tuned by the local data set on each mobile to achieve convergence and personalization. The main feature and advantage of transfer learning technology are that it relaxes two basic assumptions in traditional machine learning: (1) The training sample used for learning and the new test sample satisfy independently identical distribution. (2) A good model can only be obtained by learning with enough available training samples. There are two problems in autonomous learning: One is that it is difficult for the pre-training data to have the same distribution as the real user data; the other is that the data available for training on each mobile is limited. In the design process of AutLearn, obtaining a suitable public data set is the key for model converge on the mobiles and high accuracy. With the input method as an example, we use the corpus data set crawled from the Twitter for pre-training and use the input method data from the users on the mobile devices for transfer learning. In reality, a large part of data of Twitter is uploaded by users through mobile phones, so these data are strongly correlated in distribution and semantics, which means that the model trained by Twitter data will often learn features that include user input data. In addition, the common method of transfer learning is to fix the parameters of the front network layer, which only participates in forward inference and will not participates in backward training. Only the parameters of the last layer will change as the training progresses. This is why transfer learning can complete the training with only small samples and has low requirements for calculation power.

In order to further improve the effect of local transfer learning, AutLearn also uses data augmentation to increase the amount of training data. For image data, AutLearn uses common data augmentation techniques, such as flip, rotation, scale, crop, translation, and Gaussian noise. For natural language data, AutLearn uses the technology in the $\mathrm{EDA}^{[19]}$ open source project, which includes four main operations:

(1) Synonyms Replace (SR): Regardless of stopwords, $n$ words are randomly extracted from the sentence, and then their synonyms are randomly extracted from the synonym dictionary to replace them.

(2) Randomly Insert (RI): Regardless of stopwords, a word is randomly selected, and then one word is randomly selected from the synonym set of the word to be inserted at a random position in the original sentence. This process can be repeated for $n$ times.

(3) Randomly Swap (RS): Two words are randomly selected in the sentence, and their positions are exchanged. This process can be repeated for many times.

(4) Randomly Delete (RD): Each word in the sentence is randomly deleted with probability $p$.

Specifically, using which data augmentation techniques or combination of several techniques is determined according to the specific application scenario (classification task).

AutLearn divides the transfer learning on the mobile devices into two types according to the timing of starting training and the time generated by the training data: offline learning and online learning (Table 1). Offline learning means that on the premise of not affecting user experience (delay and quantity of electricity), training is performed at the right timing based on historical data. Offline learning usually occurs after the developer updates and releases a new model and performs transfer learning on the model to solve the cold start problem. Due to more historical data, the training is longer and cannot be guaranteed to be completed in a short time (such as one day). Therefore, AutLearn will store intermediate results and disperse the training in multiple time windows. Online learning refers to training based on data generated in real time on the devices. The main purpose of online learning is to respond to the new data timely. For example, after the user behavior changes, the model can be personalized and trained immediately, which 
is a continuously enhanced process. Although online learning will be proportionally costly to compute, because the data generated in real time is more effective, the cost caused by training is usually imperceptible. In Section 4, we mainly analyze the system resource cost caused by online learning in input method applications.

Table 1 Comparison between different on-device transfer learning in AutLearn

\begin{tabular}{llll}
\hline Type & Training data & Training timing & Advantages \\
\hline $\begin{array}{l}\text { Offline } \\
\text { learning }\end{array}$ & $\begin{array}{l}\text { Historical data saved } \\
\text { locally }\end{array}$ & $\begin{array}{l}\text { Offline idle time: } \\
\text { The phone is being } \\
\text { charged and the } \\
\text { screen is off }\end{array}$ & $\begin{array}{l}\text { - The large amount of data accumulated in } \\
\text { history; the obvious training results; can } \\
\text { effectively solve the cold start problem. } \\
\text { Offline proceeding does not affect user } \\
\text { experience, and it usually has a lot of time } \\
\text { for training. }\end{array}$ \\
$\begin{array}{llll}\text { Online } \\
\text { learning }\end{array}$ \\
$\begin{array}{llll}\text { Real-time data } \\
\text { generated by users } \\
\text { when they use the } \\
\text { device }\end{array}$ & $\begin{array}{l}\text { Online proceeding, } \\
\text { usually accompanied } \\
\text { by the user } \\
\text { interacting with the } \\
\text { device }\end{array}$ & $\begin{array}{l}\text { The data generated by users online } \\
\text { is usually slow, and using these data } \\
\text { for training has little impact on user } \\
\text { experience. } \\
\text { No need to wait for a specific training } \\
\text { timing; continuous optimization of the } \\
\text { model during the online learning process } \\
\text { to quickly improve model accuracy. }\end{array}$ \\
\end{tabular}

\subsection{Model training compiler}

There are two major ways to deploy neural networks on mobiles: One is to use general machine learning frameworks such as TensorFlow. These frameworks implement forward/backward operations of various operators which are loaded into the memory by thirdparty libraries and then are calculated after the model, and data are read in. The other is the neural network compiler represented by $\mathrm{TVM}^{[20]}$ that has emerged in recent years. These compilers generate an executable file that can be directly deployed and run on the platform for specific models and hardware platforms. For example, TVM follows and expands the concept of compute-schedule of Halide ${ }^{[21]}$, determines the code optimization process by defining the schedule, and realizes the deployment of deep learning models on various devices by automatic code generation. Compared with general machine learning frameworks, neural network compilers have the following advantages:

(1) They can automatically generate the underlying operation code according to the description of the hardware and operators and have high scalability.

(2) They can automatically search for implementations on specific hardware (including memory layout and SIMD) to achieve optimal operation efficiency. In autonomous learning scenarios, the introduction of model training compilers can effectively reduce computational cost on mobile devices.

However, the existing neural network compilers only support model inference scenarios and cannot be used for model training. Therefore, we have extended the original TVM framework to implement autonomous learning on mobiles.

As shown in Figure 4, in order to compile and generate an executable file that can be used for training, we compile the network structure and loss function input by users to obtain three networks.

(1) Runnable forward network which is used for forward operation.

(2) Runnable loss network. The difference between this network and the runnable forward network is that the loss function is added. The relevant training parameters can be obtained to determine the parameters that need to be updated through this network. 
(3) Runnable update network which is used to update the relevant parameters of the network. The relevant parameters of the network are updated to update the parameter of the calculating chart according to the input parameters, calculated relevant gradients and algorithms such as SGD.

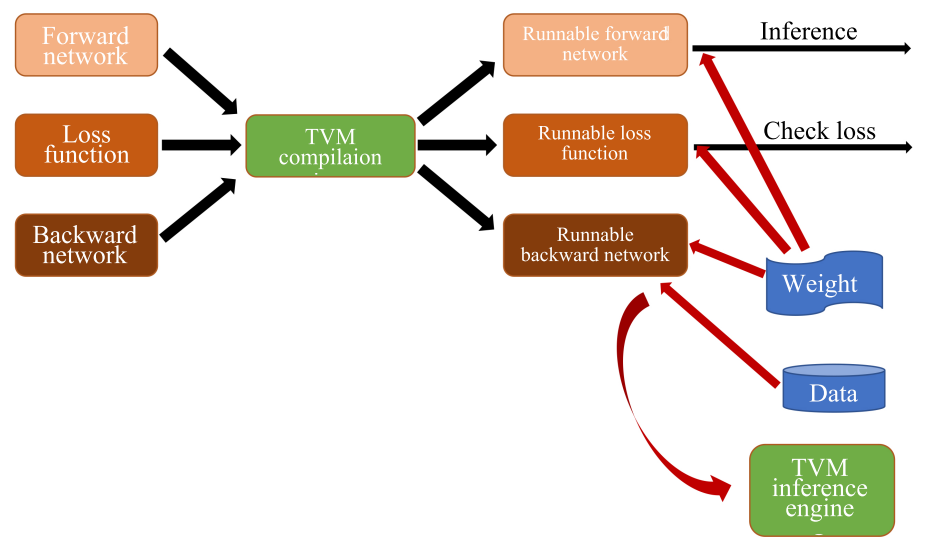

Figure 4 Deep learning compiler for autonomous learning

The purpose of generating the above three different functional networks is to make better use of the TVM modularity and reduce the modification of the TVM code base.

Finally, we supplemented the backward propagation of the missing operators in TVM, so that the binary code executed on the mobiles can be compiled for any model structure, and finally the network can be run and updated on mobile devices, namely that the training process can be realized. Backward propagation in training is basically similar to model inference in the underlying implementation. For example, the core operations are all around matrix multiplication. Therefore, in the process of compiling executable code, we follow the existing design idea of schedule in the inference process, including search space and search algorithms. More specifically, we added the search space of schedule aimed at the backward propagation operators (such as conv2d_grad and dense_grad) for the third runnable update network mentioned above which is implemented by existing template forms. As for the search algorithm, we follow the cost model based on machine learning in TVM to predict the running time of schedule, continuously update this cost model during actual compilation, and finally make the search process quickly converge.

In addition, TVM has implemented a lot of hardware-related schedule primitives inside for different terminal platforms. We design the code search space of different mobiles by these primitives and finally get the optimized code aimed at the back-end hardware after continuous testing of automatic tuning. When the expanded TVM framework presented in this paper generates training code, different mobile devices (or different hardware SoCs) are also needed for their respective tuning. During the tuning, real devices are required to offer runtime services to obtain the operation efficiency of traversing nodes in the search process as its feedback. The above search and tuning processes are needed for each device or hardware (two devices are used in the experiment in this paper), so they are time-consuming. We will try to use a more effective

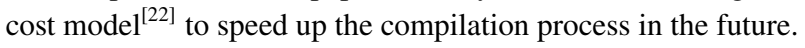

\subsection{Optimization technology of training}

Model compression ${ }^{[23]}$ is a common way to reduce the complexity of neural network models, including specific schemes such as model pruning, quantification, parameter sharing, 
and knowledge distillation. AutLearn uses a parameter pruning scheme based on magnitude ${ }^{[18]}$ : A CONVolutional layer (CONV) or a Fully Connected layer (FC) is selected in each round, and then the smallest $K$ convolution kernels of L2-norm magnitude are removed (FC can be regarded as a special CONV, which also has a convolution kernel). The value $K$ depends on the complexity of the model obtained by compressing in the end. After multiple iterations, one or more compressed models can be obtained, which are suitable for different mobile devices. In order to reduce the burden on developers, the network layers which can be pruned do not need to be selected manually, because a model automatic pruning module is built in AutLearn, namely an Automatic search algorithm of Neural network Structure (NAS) of special form, which can start from a large model to automatically generate a series of optimized models for different resource conditions. As shown in the following formula, Accuracy is used to calculate the accuracy of a model, which is measured by the validation set; Resource is used to calculate the resource consumption of a model (such as delay, energy consumption, and memory usage), which can be obtained by modeling according to the model structure ${ }^{[24,25]}$, or by prototype measurement ${ }^{[26,27]}$. This paper uses the latter to obtain more real and accurate resource data; Budget is the upper limit of available resources set by users. The resource type can be the FLOating-Point operations per second (FLOPs), the inference time, the inference energy consumption, and the memory usage of the model, which are controlled by the developer.

$$
\begin{aligned}
& \arg \max _{\text {Model }} \text { Accuracy }(\text { Model }) \\
& \text { subject to Resource }(\text { Model })<\text { Budget }
\end{aligned}
$$

At present, the pruning of AutLearn is done in the cloud, mainly because the cloud has more data to retrain the pruned model and reduce the computational load on the mobile. However, the model pruning on the mobile also has certain advantages, because the mobile data can be directly used for pruning training and testing to obtain a more accurate pruning scheme. The reason why this paper uses the pruning technology based on magnitude is that this technology has been widely proven and used, but other pruning technologies are also compatible with the AutLearn system, such as the technology in Ref. [28] which determines the optimal pruning scheme according to the influence of the pruning operation on the feature map. Compared with these more complex pruning algorithms, the pruning scheme based on the magnitude is less complex, so it is more conducive to processing on mobile devices.

In addition, the AutLearn incorporates a cache mechanism to reuse the inference results during training for online learning scenarios to reduce the cost of model training. As shown in Figure 5, the model will perform inference operations on real-time input data in online learning, generate labels according to user feedback, and form training data combined with the original data to train the model. Inference and training are performed in a pipeline system. Among them, the essence of neural network training is to calculate the loss function on the basis of the inference result and then do backward propagation. Therefore, AutLearn will reuse the predicted results and directly perform model training based on the results and the feedback behaviors (true label) provided by the users. Specifically, the data that needs to be cached in model inference is the inference prediction result (used to generate loss) and the intermediate results (namely feature map which is used for parameter update in the backward propagation process). Since the transfer learning technology is used in autonomous learning, only a few parameters at the back of the model need to be updated. It is assumed that the network layer that needs to be updated is from the $K$-th layer to the last layer, only the middle vectors generated from the $(K-1)$-th layer to the last layer need to be saved, which greatly reduces the memory cost of the runtime 
cache. In order to further reduce the performance cost of a single data, AutLearn will begin training after multiple data generation reaches a batch (the default batch size is 16 and it can be adjusted by the developer).

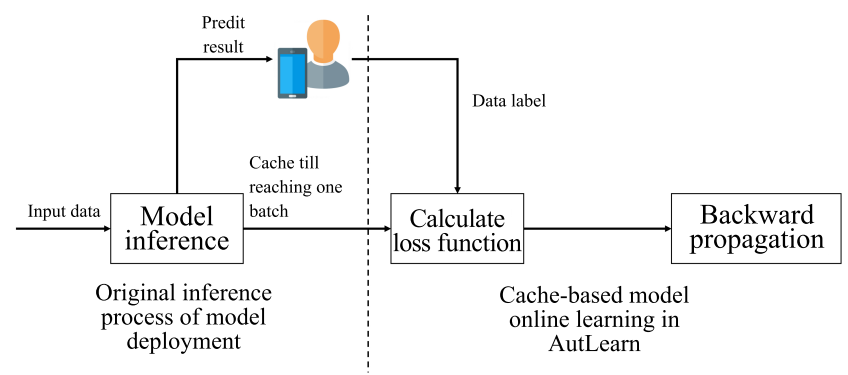

Figure 5 Workflow of inference results reuse in online learning

\subsection{Applications}

Two applications are built based on AutLearn to verify its functions and effects.

\subsubsection{Word prediction in the input method}

The input method is one of the most important applications on mobile devices. One of the main functions of the input method application is input word prediction. For example, the English input method predicts the input words that users want based on the existing input sequence typed by the user (including words and letters). The word prediction function is implemented in most mainstream input method applications. For example, the word prediction function of Gboard is realized based on neural networks and is one of the most common tasks in natural language processing tasks. One of the current mainstream algorithms for processing this task is Recurrent Neural Network (RNN). For example, the LSTM network structure which is a common variant of RNN, implements a basic neural network model of word prediction as shown in Figure 6.

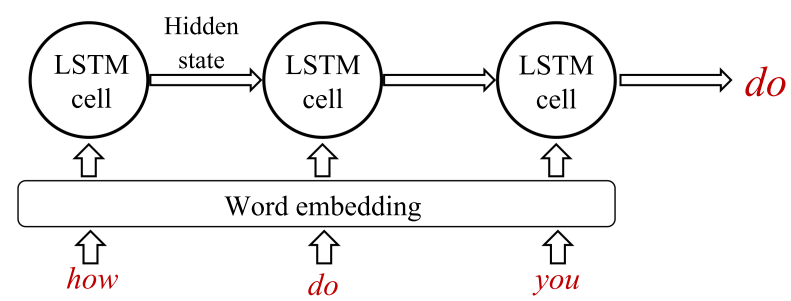

Figure 6 LSTM model structure implemented in this work

The word prediction function in the input method fits the application scenarios of AutLearn:

(1) Data privacy: User input data includes chat history and account passwords, so they should not be uploaded to a centralized server.

(2) Local generation of training data: The clicking input of users marks the data and can be used directly for training.

(3) The input behaviors of users are personalized, so the model needs to be personalized to achieve the best effect.

(4) Historical input data are usually stored locally and can be used for offline model transfer learning. 
(5) The input behaviors of users are not fixed and may change over time, so continuous learning updates (online learning) are required.

\subsubsection{Image classification}

Image processing is one of the most common application scenarios of neural networks. For example, in iPhone's inbuilt album application, convolutional neural networks have been used for recognition of objects, scenarios and faces, helping users to organize photos for easy search. Similar to the text input data in the input method, the photos taken by the user contain a large volume of private information which should not be uploaded to the server for training.

The classic convolutional neural network MobileNet is trained based on AutLearn to realize image classification on mobiles. MobileNet is a network structure specially designed for mobile devices. It internally uses structure of the depth-wise separable convolutions to reduce the calculation amount of the convolutional layer.

\section{Experiments}

This section introduces experimental design and conclusions of AutLearn. The experiments are carried out:

(1) to verify that AutLearn can obtain high accuracy for neural network models through transfer learning on mobile;

(2) to verify the hardware resources on the common mobile platform are sufficient to support the training of the neural network model, especially the effect of a series of optimization techniques on reducing the training cost of the neural network model mentioned above.

\subsection{Environment and design}

Models and data: For the two application scenarios mentioned above, different public data sets and models are used for testing.

- The word prediction application in the input method, the LSTM ${ }^{[29]}$ model is used with Twitter data as the pre-training data (public data set) and the Shakespeare data set as the local data set on each mobile device. Both data are from the standard data set of LEAF $^{[30]}$ federated learning base test and are pre-segmented for different users. The data of different users is non-iid. Both data sets are used for prediction training of input words. In transfer learning, only the last fully connected layer participates in the model updating. The specific parameters of the LSTM model used in this paper are the vocabulary composed of 10,000 most frequently used words, and Step Size is the size of variable length with two-layer stacked LSTM Cell implemented by the bucketing technology ${ }^{[31]}$.

- In the image classification application, the MobileNet ${ }^{[32]}$ model is used with the ImageNet data set as the pre-training data and the FEMINST data set as the local data set on each mobile device. Among them, FEMINST is also from the standard data set of the original LEAF, and the data are non-iid. ImageNet dataset is used to train object recognition of 1,000 categories of MobileNet, and FEMINST is used for transfer learning and handwriting recognition tasks. In transfer learning, only the last fully connected layer and the Softmax layer will participate in the model updating. Only the $10 \%$ of users with the largest volume of data are selected from two data sets for training and testing, because the accumulation of historical data of users is often larger than the data volume of standard data sets in reality.

Baseline: AutLearn is compared with the following solutions: 
- Federated learning is the technology that has emerged in recent years for model training on distributed data (Section 1). The most common federated learning algorithm FedAvg ${ }^{[3]}$ is used here. Compared with autonomous learning technology, federated learning does not personalize the models of different users and requires huge communication cost.

- Centralized cloud training technology (CloudTrain) ${ }^{[33]}$ collects user data to each mobile, and then uses these data to train a general model. Similarly, CloudTrain does not personalize the model training. The centralized training mode is also the universal deployment method in the industry now.

- Centralized personalized training technology (CloudCustomize $)^{[34]}$ refers to the results of personalized training of the data of the new model on different devices based on the model obtained by CloudTrain. On accuracy grounds, CloudCustomize can be considered as the optimal situation, because it adopts user private data for pre-training and transfer learning. However, the CloudCustomize has defects in user privacy and scalability.

AutLearn and all baselines perform model training by mini-batch ${ }^{[35]}$.

Mobile device: Samsung Note 8 and Redmi Note 8 are used to test the training efficiency of AutLearn on mobile devices, including trainable time and energy consumption.

\subsection{Model accuracy}

In Figure 7, the accuracy comparison of AutLearn under different models and each baseline is summarized in offline learning. The results show that AutLearn and each baseline can obtain a convergent model by training. Compared with the traditional training mode such as CloudTrain, the model obtained by FedAvg federated learning training mode has a small loss of accuracy (about 1\%), while the accuracy of AutLearn is basically the same as or even higher than that of CloudTrain mode: AutLearn has a $0.9 \%$ accuracy decrease compared to CloudTrain on LSTM model, while a $0.8 \%$ accuracy increase on MobileNet model. The reason is that AutLearn adopts an autonomous learning method, namely each user model is trained by the data generated by the specific user to achieve the effect of personalization in the process of on-device transfer learning. In addition, CloudCustomize method achieves the highest accuracy on both models, because it performs personalized training on the basis of CloudTrain centralized training, and uses the same user private data set. However, AutLearn performs cloud pre-training using data sets with different distributions to obtain a global model, and then performs a transfer learning using a data set with the same source as the test data set. Because pre-training and transfer learning use different data sets, the feature extraction that the model learns in pre-training may not be completely applicable to user private data. As a result, the effect of AutLearn mode is not as good as that of the CloudCustomize mode. Among the four training modes, only FedAvg and AutLearn consider user privacy protection. In the series of experiments, it is found that the effect of model personalization is limited because the data set limits the amount of data on each device.

The impact of data augmentation technology on the accuracy of the model is verified. For the LSTM model, the synonym replacement technology is used. For the MobileNet model, cropping, rotation, and shifting techniques are used. Both techniques can increase the training data volume by more than 5 times. The data of the experiments are sampled from the generated data and their volume does not exceed the original data volume to balance the computational cost of training. The results show that data augmentation improves the accuracy of $0.9 \%$ and $3.1 \%$ for word prediction and image classification, respectively. The latter has a more obvious effect: In fact, data augmentation in natural language processing is indeed more difficult than image data.

In addition, the effect of increasing model accuracy of online learning is explored through 
experiments. Starting from the pre-trained model, the data set is then serialized and continuously input into the AutLearn system. For each piece of data, AutLearn will first make prediction, and then perform intensive model training by using the data and repeat the process, and finally summarize the overall results of the prediction. This process simulates the adaptation of AutLearn to the user current behaviors (data generation) when users are using the model. It is found that for the LSTM and MobileNet models, compared to only use the pre-trained model to predict all data, online learning can increase the model accuracy of $5.9 \%$ and $3.1 \%$ on all data on average. The increase of the later input data are more obvious, because as more data are input, the model gets continuous learning and the accuracy increases. The results show that online learning can adjust the model adaptively and effectively according to the changes of user behaviors (such as input patterns) to achieve high accuracy.

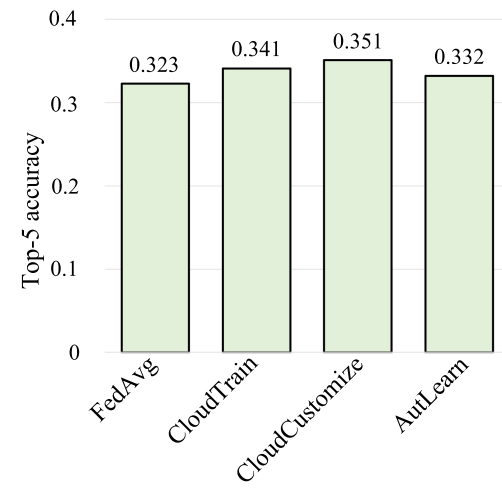

(a) LSTM model

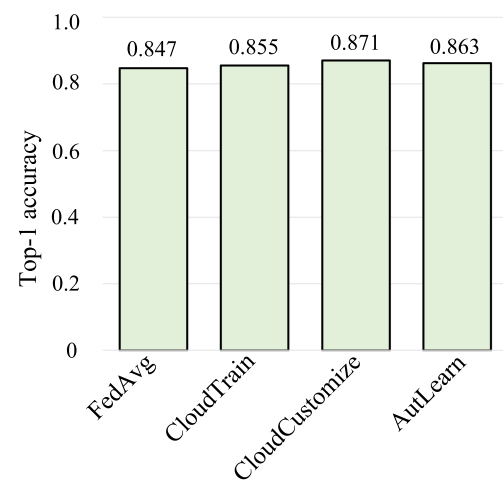

(b) MobileNet model

Figure 7 Accuracy of AutLearn compared with baselines

\subsection{Mobile training cost}

We test the performance cost of transfer learning (offline learning) on two end devices, and the results are shown in Figure 8. AutLearn represents the training speed of offline learning; w/o compression and w/o compiler denote the performance when the model compression and compiler acceleration techniques mentioned above are removed respectively, and w/o cache is the online trainable time accelerated by the inference-training cache. "w/o compiler" means using a generic deep learning library to train model (TensorFlow). For the LSTM model, we used batch size 16 for training, while for the MobileNet model, due to the large memory occupation, only batch size 1 could be used for training on experimental mobiles.

The results are as follows:

(1) Model compression technique can effectively shorten the trainable time, but the performance of diverse models is very different. The model can be effectively compressed with only $1 \%$ accuracy loss. Model compression technology can reduce trainable time by more than $80 \%$ on the LSTM model, but only $20 \%-30 \%$ on the MobileNet model. The main reason is that MobileNet is a very compact model designed for devices with low computing power, which makes compression more difficult.

(2) Compiler technology is also effective in reducing trainable time, saving nearly $40 \%$ on both the LSTM and the MobileNet models. Compared with model compression, the compiler technology basically does not depend on the structure of the model itself, and not cause the loss of model accuracy. 
(3) The inference-training caching mechanisms used in online learning can further reduce trainable time $(30 \%-40 \%)$ of the model, because the forward inference process of model training is saved, and only the backward inference is needed. The caching mechanism is only effective in online learning, because parameters will be updated after each batch training in offline study, causing the original predictions of caches to fail on the new model and cannot be reused.

(4) The trainable time on mobile devices is up to nearly twice different, because computational resources on diverse mobile devices vary. However, the performance of optimization technology of AutLearn is improved on different devices and the optimization technology is universally applicable.

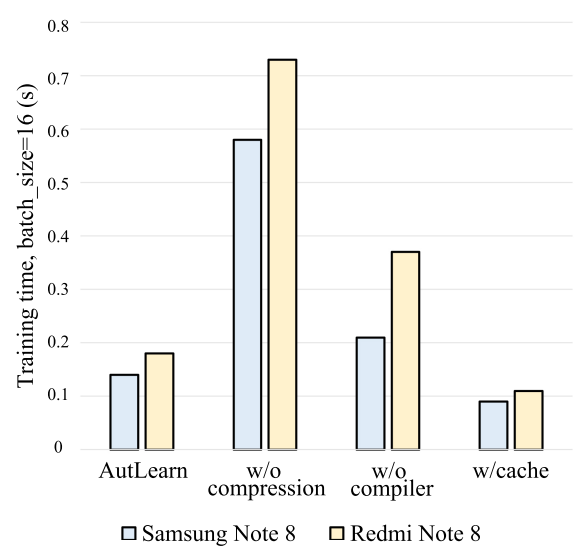

(a) LSTM model

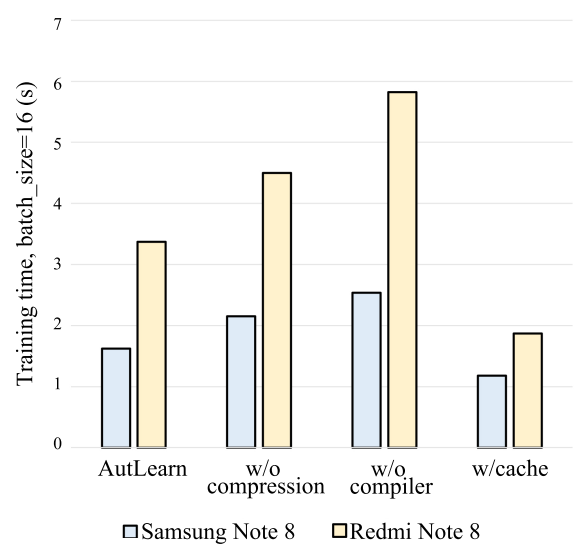

(b) MobileNet model

Figure 8 Performance of AutLearn on different device models (including online/offline learning)

Combined with the analysis of the behavior data of real users mentioned in Section 2, it is found that more than half of the mobiles are trained for about 120 minutes a day, which means that both the LSTM model and the MobileNet model can complete transfer learning training for several rounds within one day, namely that the new model can be deployed on the second day after being issued and achieve a high accuracy after personalization.

The energy consumption of AutLearn on the mobile device is measured. With the Samsung Note 8 as an example, as shown in Figure 9, "interactive" and "power save" represent different CPU status regulators. The former will keep the CPU running at a higher frequency (the default regulator of Android devices), while the latter will keep the CPU running at the lowest frequency to reduce energy consumption. All results are normalized to the same baseline: (1) Optimization acceleration technology of AutLearn is not used and CPU is in operation under the interactive regulator. According to the results, the optimization technology of AutLearn can greatly reduce the energy consumption of mobile model training by up to more than $80 \%$ (LSTM model), mainly because the mobile-to-mobile time of model training is saved. (2) Under the low-frequency running status of CPU, although the training of the model takes more time, the overall energy consumption has been decreased, because the energy consumption of CPU per unit time is much lower in the low-frequency running status. This means that in some scenarios where model training does not need to be completed quickly for deployment, the CPU frequency can be lowered to cut energy consumption. 


\section{Conclusions}

In this paper, an autonomous machine learning model is proposed to solve the problem of data privacy for mobile terminal. Different from the traditional centralized and federal methods, autonomous learning deploys all the computations related to private data locally, which greatly improves the ability of protecting user privacy and provides the model personalization. In order to solve the two challenges of insufficient data and computing power on mobile devices in autonomous learning, we designed and implemented the AutLearn autonomous learning framework, which includes the cloud-client cooperation training, local data augmentation, model compression, and cache reuse. With classic natural language processing and image recognition tasks as examples, the effect of autonomous learning is verified on real datasets: AutLearn can achieve similar or even higher accuracy than traditional training modes, while its computational cost is within the range of a typical smartphone.

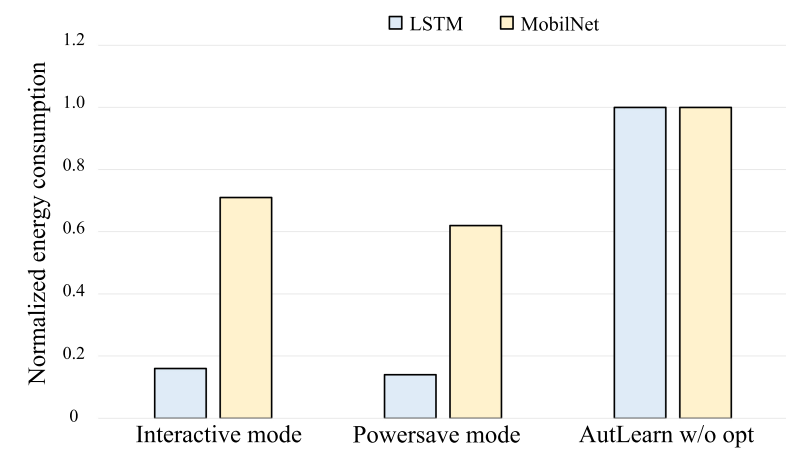

Figure 9 Energy consumption of AutLearnin different CPU statuses

\section{References}

[1] Xu M, Liu J, Liu Y, Lin FX, Liu Y, Liu X. A first look at deep learning apps on smartphones. Proc. of the World Wide Web Conf. 2019. 2125-2136.

[2] General Data Protection Regulation (GDPR). 2018. https://gdpr-info.eu/

[3] McMahan HB, Moore E, Ramage D, Hampson S. Communication-efficient learning of deep networks from decentralized data. arXiv Preprint arXiv: 1602.05629, 2016.

[4] Bost R, Popa RA, Tu S, Goldwasser S. Machine learning classification over encrypted data. Proc. of the NDSS. 2015. 4324-4325.

[5] Abadi M, Chu A, Goodfellow I, McMahan HB, Mironov I, Talwar K, Zhang L. Deep learning with differential privacy. Proc. of the 2016 ACM SIGSAC Conf. on Computer and Communications Security. 2016. 308-318.

[6] Konečný J, McMahan HB, Ramage D, Richtárik P. Federated optimization: Distributed machine learning for on-device intelligence. arXiv Preprint arXiv: 1610.02527, 2016.

[7] Li L, Xiong H, Wang J, Xu CZ, Guo Z. SmartPC: Hierarchical pace control in real-time federated learning system. Proc. of the IEEE Real-time Systems Symp. (RTSS 2019). 2019.

[8] Bonawitz K, Ivanov V, Kreuter B, Marcedone A, McMahan HB, Patel S, Ramage D, Segal A, Seth K. Practical secure aggregation for privacy-preserving machine learning. Proc. of the 2017 ACM SIGSAC Conf. on Computer and Communications Security. 2017. 1175-1191.

[9] Vaidya J, Kantarcioğlu M, Clifton C. Privacy-preserving naive Bayes classification. The VLDB Journal, 2008, 17(4): 879-898.

[10] Graepel T, Lauter K, Naehrig M. ML confidential: Machine learning on encrypted data. Proc. of the 
Int'l Conf. on Information Security and Cryptology 2012. Berlin, Heidelberg: Springer-Verlag, 2012. $1-21$.

[11] Nandakumar K, Ratha N, Pankanti S, Halevi S. Towards deep neural network training on encrypted data. Proc. of the IEEE Conf. on Computer Vision and Pattern Recognition Workshops. 2019.

[12] Xiong P, Zhu T, Wang X. A Survey on differential privacy and applications. Chinese Journal of Computers, 2014, 37(1): 101-122 (in Chinese with English abstract).

[13] Shokri R, Shmatikov V. Privacy-preserving deep learning. Proc. of the 22nd ACM SIGSAC Conf. on Computer and Communications Security. 2015. 1310-1321.

[14] Bagdasaryan E, Veit A, Hua Y, Estrin D, Shmatikov V. How to backdoor federated learning. arXiv Preprint arXiv: 1807.00459, 2018.

[15] Chellappa RK, Sin RG. Personalization versus privacy: An empirical examination of the online consumer's dilemma. Information Technology and Management, 2005, 6(2/3): 181-202.

[16] Bonawitz K, Eichner H, Grieskamp W, Huba D, Ingerman A, Ivanov V, Kiddon C, Konecny J, Mazzocchi S, McMahan HB, Van Overveldt T. Towards federated learning at scale: System design. Proc. of the SysML. 2019.

[17] Zhuang F, Luo P, He Q, Shi Z. Survey on transfer learning research. Ruan Jian Xue Bao/Journal of Software, 2015, 26(1): 26-39. http://www.jos.org.cn/1000-9825/4631.htm [doi: 10.13328/j.cnki.jos.004631] (in Chinese with English abstract).

[18] Xue J, Li J, Gong Y. Restructuring of deep neural network acoustic models with singular value decomposition. Proc. of the Interspeech. 2013. 2365-2369.

[19] Wei JW, Zou K. EDA: Easy data augmentation techniques for boosting performance on text classification tasks. Proc. of the EMNLP/IJCNLP (1). 2019. 6381-6387.

[20] Chen T, Moreau T, Jiang Z, Zheng L, Yan E, Shen H, Cowan M, Wang L, Hu Y, Ceze L, Guestrin C. TVM: An automated end-to-end optimizing compiler for deep learning. Proc. of the 13th USENIX Symp. on Operating Systems Design and Implementation (OSDI 18). 2018. 578-594.

[21] Ragan-Kelley J, Barnes C, Adams A, Paris S, Durand F, Amarasinghe S. Halide: A language and compiler for optimizing parallelism, locality, and recomputation in image processing pipelines. ACM SIGPLAN Notices, 2013, 48(6): 519-530.

[22] Zerrell T, Bruestle J. Stripe: Tensor compilation via the nested polyhedral model. arXiv Preprint arXiv: 1903.06498, 2019.

[23] Lei J, Gao X, Song J, Wang XL, Song ML. Survey of deep neural network model compression. Ruan Jian Xue Bao/Journal of Software, 2018, 29(2): 251-266. http://wwww.jos.org.cn/1000-9825/5428. htm [doi: 10.13328/j. cnki.jos.005428]

[24] Xu M, Qian F, Zhu M, Huang F, Pushp S, Liu X. Deepwear: Adaptive local offloading for on-wearable deep learning. IEEE Trans. on Mobile Computing, 2019, 19(2): 314-330.

[25] Xu M, Zhu M, Liu Y, Lin FX, Liu X. DeepCache: Principled cache for mobile deep vision. Proc. of the 24th Annual Int'1 Conf. (MobiCom). 2018. 129-144.

[26] Yang TJ, Howard A, Chen B, Zhang X, Go A, Sandler M, Sze V, Adam H. Netadapt: Platform-aware neural network adaptation for mobile applications. Proc. of the European Conf. on Computer Vision (ECCV). 2018. 285-300.

[27] Tan M, Chen B, Pang R, Vasudevan V, Sandler M, Howard A, Le QV. MNASnet: Platform-aware neural architecture search for mobile. Proc. of the IEEE Conf. on Computer Vision and Pattern Recognition. 2019. 2820-2828.

[28] Yang TJ, Chen YH, Sze V. Designing energy-efficient convolutional neural networks using energy-aware pruning. Proc. of the IEEE Conf. on Computer Vision and Pattern Recognition. 2017. 5687-5695.

[29] Hochreiter S, Schmidhuber J. Long short-term memory. Neural Computation, 1997, 9(8): 1735-1780.

[30] Caldas S, Wu P, Li T, Konečný J, McMahan HB, Smith V, Talwalkar A. Leaf: A benchmark for federated settings. arXiv Preprint arXiv: 1812.01097, 2018.

[31] Khomenko V, Shyshkov O, Radyvonenko O, Bokhan K. Accelerating recurrent neural network training 
using sequence bucketing and multi-GPU data parallelization. Proc. of the 1st IEEE Int'l Conf. on Data Stream Mining \& Processing (DSMP). 2016. 100-103.

[32] Howard AG, Zhu M, Chen B, Kalenichenko D, Wang W, Weyand T, Andreetto M, Adam H. Mobilenets: Efficient convolutional neural networks for mobile vision applications. arXiv Preprint arXiv: 1704.04861, 2017.

[33] Krizhevsky A, Sutskever I, Hinton GE. Imagenet classification with deep convolutional neural networks. Advances in Neural Information Processing Systems. 2012. 1097-1105.

[34] Taylor SA, Jaques N, Nosakhare E, Sano A, Picard R. Personalized multitask learning for predicting tomorrow's mood, stress, and health. IEEE Trans. on Affective Computing, 2017.

[35] Li M, Zhang T, Chen Y, Smola AJ. Efficient mini-batch training for stochastic optimization. Proc. of the 20th ACM SIGKDD Int'l Conf. on Knowledge Discovery and Data Mining. 2014. 661-670.

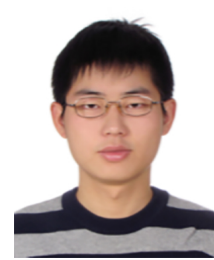

Mengwei Xu, Ph.D. His research interests include mobile/edge computing and operating system.

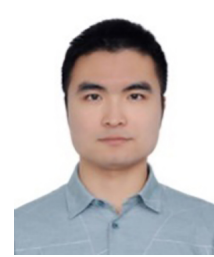

Yuanqiang Liu, master. His research interests include mobile/edge computing and operating system.

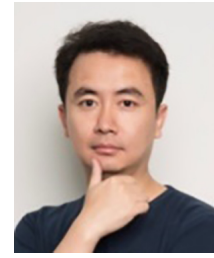

Kang Huang, Ph.D. His research interests include machine learning and natural language processing.

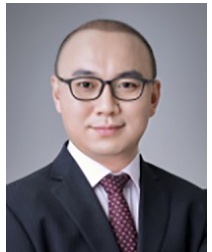

Xuanzhe Liu, Ph.D., associate professor, doctoral supervisor, CCF professional member. His research interests include service computing, Web technology, and software engineering.

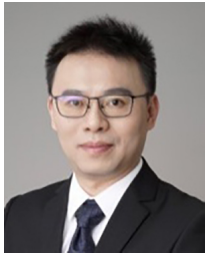

Gang Huang, Ph.D., doctoral supervisor, CCF senior member. His research interests include software middleware, software architecture and internetware. 